\title{
Catabolite repression of phosphoenolpyruvate carboxykinase by a zinc finger protein under biotin- and pyruvate carboxylase-deficient conditions in Pichia pastoris
}

\author{
Correspondence \\ Pundi N. Rangarajan \\ pnr@biochem.iisc.ernet.in
}

Received 20 July 2011

Revised 5 September 2011

Accepted 17 September 2011

\author{
Nallani Vijay Kumar and Pundi N. Rangarajan \\ Department of Biochemistry, Indian Institute of Science, Bangalore 560012, India
}

\begin{abstract}
We have identified a methanol- and biotin-starvation-inducible zinc finger protein named ROP [repressor of phosphoenolpyruvate carboxykinase (PEPCK)] in the methylotrophic yeast Pichia pastoris. When $P$. pastoris strain GS115 (wild-type, WT) is cultured in biotin-deficient, glucoseammonium ( $\mathrm{Bio}^{-}$) medium, growth is suppressed due to the inhibition of anaplerotic synthesis of oxaloacetate, catalysed by the biotin-dependent enzyme pyruvate carboxylase (PC). Deletion of $R O P$ results in a strain $(\triangle R O P)$ that can grow under biotin-deficient conditions due to derepression of a biotin- and PC-independent pathway of anaplerotic synthesis of oxaloacetate. Northern analysis as well as microarray expression profiling of RNA isolated from WT and $\triangle R O P$ strains cultured in $\mathrm{Bio}^{-}$medium indicate that expression of the phosphoenolpyruvate carboxykinase gene (PEPCK) is induced in $\triangle R O P$ during biotin- or PC-deficiency even under glucose-abundant conditions. There is an excellent correlation between $P E P C K$ expression and growth of $\triangle R O P$ in $\mathrm{Bio}^{-}$medium, suggesting that ROP-mediated regulation of PEPCK may have a crucial role in the biotin- and PC-independent growth of the $\triangle R O P$ strain. To our knowledge, ROP is the first example of a zinc finger transcription factor involved in the catabolite repression of PEPCK in yeast cells cultured under biotin- or PC-deficient and glucose-abundant conditions.
\end{abstract}

\section{INTRODUCTION}

Biotin, a water-soluble vitamin (vitamin $\mathrm{H}$ ) is an essential nutrient for the growth of several laboratory strains of Saccharomyces cerevisiae as well as the methylotrophic yeast Pichia pastoris. It functions as the prosthetic group of several carboxylases involved in anaplerosis, fatty acid biosynthesis, gluconeogenesis and amino acid metabolism. In S. cerevisiae, biotin is essential for the catalytic activity of pyruvate carboxylase (PC), acetyl-CoA carboxylase, urea amidohydrolase and mitochondrial Hfalp (Pirner \& Stolz, 2006). Arclp, a protein involved in cytosolic confinement of amino acyl tRNA synthetases, is also biotinylated (Kim et al., 2004). Many laboratory strains of S. cerevisiae such as S288c possess only a partial biotin biosynthetic pathway and therefore cannot synthesize biotin de novo. However, biotin-prototrophic strains of $S$. cerevisiae have been reported (Phalip et al., 1999; Wu et al., 2005). P. pastoris

Abbreviations: ICL, isocitrate lyase; MS, malate synthase; Mut, methanol utilization; OAA, oxaloacetate; PC, pyruvate carboxylase; PEP, phosphoenolpyruvate; PEPCK, phosphoenolpyruvate carboxykinase.

The GEO accession number for the microarray data associated with this paper is GSE32948.

Three supplementary figures are available with the online version of this paper. strain GS115 (wild-type, WT) does not possess a de novo biotin biosynthetic pathway. However, expression of four S. cerevisiae genes involved in biotin biosynthesis resulted in a $P$. pastoris strain capable of de novo biotin synthesis (Gasser et al., 2010).

In general, yeast cells cannot grow in a biotin-deficient medium $\left(\mathrm{Bio}^{-}\right)$containing glucose and ammonium as sole sources of carbon and nitrogen respectively. Growth inhibition is primarily due to the non-replenishment of the citric acid cycle intermediate oxaloacetate (OAA) by the biotin-dependent enzyme PC. When such media are supplemented with aspartate, growth is restored due to replenishment of OAA via transamination of $\alpha$-ketoglutarate (Zelle et al., 2010; Blázquez et al., 1995). OAA can be synthesized in a PC- and biotin-independent manner via the glyoxylate cycle when yeast cells are cultured in media containing non-fermentative carbon sources such as acetate. However, enzymes of the glyoxylate cycle are repressed under glucose-abundant conditions (Blázquez et al., 1995). In $S$. cerevisiae, strains carrying mutations in the genes encoding PC and pyruvate kinase, phosphoenolpyruvate carboxykinase (PEPCK), which normally has decarboxylating and gluconeogenic functions, can replace the anaplerotic function of PC in glucose-grown cultures (Zelle et al., 2010). While PC-deficient yeast cells cannot grow in a medium 
containing glucose and ammonium as the sole sources of carbon and nitrogen respectively, certain mutations termed $B P C$ (bypass of pyruvate carboxylase) enable them to grow under these conditions (Blázquez et al., 1995). BPC mutants synthesize OAA by a PC-independent mechanism involving either derepression of enzymes of the glyoxylate pathway or by other mechanisms that bypass PC function (Blázquez et al., 1995). However, the transcription factors responsible for the $B P C$ phenotype are not known.

Mxrlp is a zinc finger protein that plays a key role in the regulation of genes of the methanol utilization (Mut) pathway as well as those encoding peroxisomal enzymes in P. pastoris (Lin-Cereghino et al., 2006). Mxrlp regulates the expression of target genes by binding to MXR response elements (MXREs) comprising of a core 5'-CYCCNY-3' motif (Kranthi et al., 2009, 2010). The P. pastoris genome encodes a large number of zinc finger proteins, many of which are still not well characterized (De Schutter et al., 2009). In this study, we have characterized a novel methanolinducible zinc finger protein named ROP which has a DNAbinding domain similar to that of Mxrlp but with a different biological function. In addition to methanol, $R O P$ expression is also induced in $P$. pastoris cells by biotin deficiency and ROP regulates the expression of several genes including that encoding the gluconeogenic enzyme PEPCK.

\section{METHODS}

Media, yeast strains and yeast culture techniques. $P$. pastoris strain GS115 (his4) was cultured at $30{ }^{\circ} \mathrm{C}$ in shake flasks containing YPD medium ( $1 \%$ yeast extract, $2 \%$ peptone and $2 \%$ glucose) or YNB medium $(0.67 \%$ yeast nitrogen base without amino acids) supplemented with $50 \mu \mathrm{g}$ histidine $\mathrm{ml}^{-1}$ and either $2 \%$ glucose (YNBD), $2 \%(\mathrm{v} / \mathrm{v})$ glycerol (YNBG), $2 \%$ methanol (YNBM), or $0.5 \%$ oleic acid and $0.05 \%$ Tween 40 (YNBO). For studying the effect of biotin, $P$. pastoris cells were grown in a synthetic mineral (SM) medium containing $\left(\mathrm{Bio}^{+}\right)$or lacking $\left(\mathrm{Bio}^{-}\right)$biotin. One litre of SM contained $20 \mathrm{~g}$ glucose, $4 \mathrm{mg}$ L-histidine, $200 \mathrm{ml} 5 \times$ salts, $10 \mathrm{ml}$ $100 \times$ vitamin mixture and $10 \mathrm{ml} 100 \times$ trace element mixture. The $5 \times$ salt solution was prepared by dissolving $5 \mathrm{~g}\left(\mathrm{NH}_{4}\right) \mathrm{H}_{2} \mathrm{PO}_{4}, 1.12 \mathrm{~g}$ $\mathrm{MgSO}_{4}, 1.23 \mathrm{~g} \mathrm{KCl}$ and $125 \mathrm{mg} \mathrm{NaCl}$ in $100 \mathrm{ml}$ water. A $100 \mathrm{ml}$ solution of $100 \times$ vitamin mixture consisted of $1 \mathrm{~g}$ thiamine hydrochloride and $3 \mathrm{mg}$ biotin. A $100 \mathrm{ml}$ preparation of $100 \times$ trace element mixture contained $1 \mathrm{mg} \mathrm{H}_{3} \mathrm{BO}_{3}, 1 \mathrm{mg} \mathrm{NiSO}$, $1 \mathrm{mg} \mathrm{KI}, 1 \mathrm{mg}$ $\mathrm{Na}_{2} \mathrm{MoO}_{4} \cdot 2 \mathrm{H}_{2} \mathrm{O}, 1 \mathrm{mg} \mathrm{CoCl} 2,0.5 \mathrm{~g} \mathrm{Fe}\left(\mathrm{NH}_{4}\right)\left(\mathrm{SO}_{4}\right)_{2}, 40 \mathrm{mg} \mathrm{CuSO}$, $150 \mathrm{mg} \mathrm{ZnSO} \mathrm{Zn}_{4} .7 \mathrm{H}_{2} \mathrm{O}, 240 \mathrm{mg} \mathrm{MnSO}_{4} \cdot \mathrm{H}_{2} \mathrm{O}$ and $0.5 \mathrm{~g}$ EDTA. During the preparation of $\mathrm{Bio}^{-}$medium, biotin was omitted and avidin $\left(23 \mu \mathrm{g} \mathrm{ml}^{-1}\right)$ was added to remove trace amounts of biotin present in the medium. In some experiments, phenyl acetic acid $(2.5 \mathrm{mM})$ was added to $\mathrm{Bio}^{+}$media to inhibit PC.

P. pastoris was transformed with DNA by electroporation (Gene Pulser Xcell Electroporation System, Bio-Rad) as per the manufacturer's instructions. Zeocin-resistant colonies were selected on YPD plates containing zeocin $\left(100 \mu \mathrm{g} \mathrm{ml}^{-1}\right)$. For the study of $R O P$ expression in $P$. pastoris cultured with different carbon sources, cells were first grown overnight in YPD medium to stationary phase. An aliquot of the stationary culture was used to inoculate YNBD, YNBM, YNBG or YNBO medium at $\mathrm{OD}_{600} \sim 0.5$ and cells were grown for 12-24 h.

Nucleic acid isolation, Northern and Southern blot analysis. Total RNA was isolated from $P$. pastoris cells by the hot-phenol method (Schmitt et al., 1990). Electrophoresis of RNA on formaldehyde-agarose gels and Northern analysis was carried out essentially as described by Maniatis et al. (1982). Isolation of P. pastoris genomic DNA, random-primed labelling of cDNAs with Klenow polymerase and $\left[\alpha_{-}{ }^{32} \mathrm{P}\right] \mathrm{dCTP}$, and Southern blotting, were carried out using standard protocols (Maniatis et al., 1982). The cDNA probes used for Northern and Southern analysis were obtained by PCR amplification of $P$. pastoris genomic DNA using the following gene-specific primers: AOXI (alcohol oxidase I), 5' -atggctatccccgaagagtttg- $3^{\prime}$ and $5^{\prime}$-aagccctcaagaagtcctggca-3'; FLDI (formaldehyde dehydrogenase I), 5'-atgtctaccgaaggtcaaatcatc- $3^{\prime}$ and $5^{\prime}$-ggagcttcgtcttggactttaacc- $3^{\prime} ;$ DHAS (dihydroxyacetone synthase), $5^{\prime}$-atggctagaattccaaaagcagtatc- $3^{\prime}$ and $5^{\prime}$ ccaacaatagcatagatagtgttgtc- $3^{\prime} ;$ PEX8 (peroxin 8 ), $5^{\prime}$-atgtatagattgggatctcagggaag- $3^{\prime}$ and $5^{\prime}$-acgtcatctcttcctttgatgctgag- $3^{\prime}$; PEX14 (peroxin 14), $5^{\prime}$-atgtccagtatacgtgaagaaat- $3^{\prime}$ and $5^{\prime}$-atgactttataaagcccaaacgaaac- $3^{\prime}$, BIO2 (biotin synthase), $5^{\prime}$-atgaacagacagtcgttgatggcagg- $3^{\prime}$ and $5^{\prime}$-ctttctggtgctaacagttctgtatg- $3^{\prime}$; BPL1 (biotin-apoprotein ligase), $5^{\prime}$-atgcaactccgaaacctggtagac- $3^{\prime}$ and $5^{\prime}$-acagtcctaccacatccaggtagc- $3^{\prime}$; GAPDH (glyceraldehyde-3phosphate dehydrogenase), $5^{\prime}$-atggctatcactgtcggtattaac- $3^{\prime}$ and $5^{\prime}$-gaaagtgtcgttgacaaccttggc- $3^{\prime}$; PEPCK (phosphoenolpyruvate carboxykinase), $5^{\prime}$-atggctcctactgctatagatttac- $3^{\prime}$ and $5^{\prime}$-ttcctcagtagggcgaatcaacatg- $3^{\prime}$; ICL (isocitrate lyase), $5^{\prime}$-atgtcaggaccttacactaagattgac- $3^{\prime}$ and $5^{\prime}$-gaagtcagtgtaaggcaacttagctc- $3^{\prime} ; M S$ (malate synthase), $5^{\prime}$-atgacaccccagcaaacgtatataaac- $3^{\prime}$ and $5^{\prime}$-taacttgtccgtaaataatattagacc- $3^{\prime} ; R O P, 5^{\prime}$-cgcggatccatgtcgtcgtcatcttcc- $3^{\prime}$ and $5^{\prime}$-cccaagctttcaaccaaccgtttgtggcag- $3^{\prime}$.

Disruption of ROP and generation of the $\triangle R O P$ strain. The $\triangle R O P$ strain was generated by replacing $2685 \mathrm{bp}$ of $R O P$ coding region ( +1 to $+2685 \mathrm{bp}$ ) with a zeocin expression cassette. The $R O P$ knockout construct consisting of a zeocin expression cassette flanked by $930 \mathrm{bp}$ of $R O P$ promoter at the $5^{\prime}$ end and $930 \mathrm{bp}$ of ROP $3^{\prime}$ untranslated region at the $3^{\prime}$ end was generated by four different PCRs using P. pastoris genomic DNA and pGAPZA vector (Invitrogen) as templates as well as a series of overlapping and non-overlapping primers. To begin with, $930 \mathrm{bp}$ of $R O P$ promoter (from position -930 to -1 ) was amplified from $P$. pastoris genomic DNA using primers $5^{\prime}$ gctaatgtagtcattgattaccgcttggaaac-3' (1F: positions -930 to -898 of $R O P$ promoter) and 5'-AGCTATGGTGTGTGGGGGATCCGCAtcttgggtttgtttcacaacgcaaa-3' [1R: nucleotides 986-962 of pGAPZA (upper case); nucleotides -1 to -25 of $R O P$ promoter (lower case)]. In another PCR, a $1.17 \mathrm{~kb}$ region of the PGAPZA vector (between positions 1419 and 2591) comprising the TEF1 and EM7 promoters, zeocin resistance gene (ShBle) and CYC1 transcription termination region was amplified using the primer pair 5'-tttgcgttgtgaaacaaacccaagaTGCGGATCCCCCACACACCATAGCT $-3^{\prime}$ [2F: positions -25 to -1 of the ROP promoter (lower case); positions 962-986 of pGAPZA (upper case)] and 5'gtaaatgcatagtcattatttaccTGCTCACATGTTGGTCTCCAGCTTG-3' [2R: positions 2710 to 2686 of ROP $3^{\prime}$ untranslated region (lower case); nucleotides 2160-2136 of pGAPZA (upper case)]. In the third PCR, $930 \mathrm{bp}$ of the $3^{\prime}$ untranslated region of ROP was amplified from $P$. pastoris genomic DNA using the primer pair $5^{\prime}$-CAAGCTGGAGACGAACATGTGAGCAggtaaaataatgactatgcatttac-3' [3F: nucleotides 2136-2160 of pGAPZA (upper case); nucleotides 2686-2710 of ROP (lower case)] and $5^{\prime}$-aatggtacgatggagtgtcttcagaagaag- $3^{\prime}$ (3R: nucleotides 3616-3586 of ROP). Finally, the three PCR products containing overlapping regions were pooled and used as templates in a PCR along with primers $1 \mathrm{~F}$ and $3 \mathrm{R}$. The final $3.06 \mathrm{~kb}$ PCR product thus obtained was used to transform $P$. pastoris strain GS115 to generate the zeocinresistant $\triangle R O P$ strain.

Gene expression profiling using $\boldsymbol{P}$. pastoris microarray. A custom $P$. pastoris $8 \times 15 \mathrm{~K}$ microarray (Amadid no: 025088) was designed by Genotypic Technology (http://www.genotypic.co.in) based on the annotated $P$. pastoris genome sequence (Taxonomy ID: 644223) available in GenBank. Total RNA was isolated from $P$. pastoris cells by the hot-phenol method (Schmitt et al., 1990) and RNA integrity was assessed using an RNA 6000 Nano Lab Chip on the 
(a)

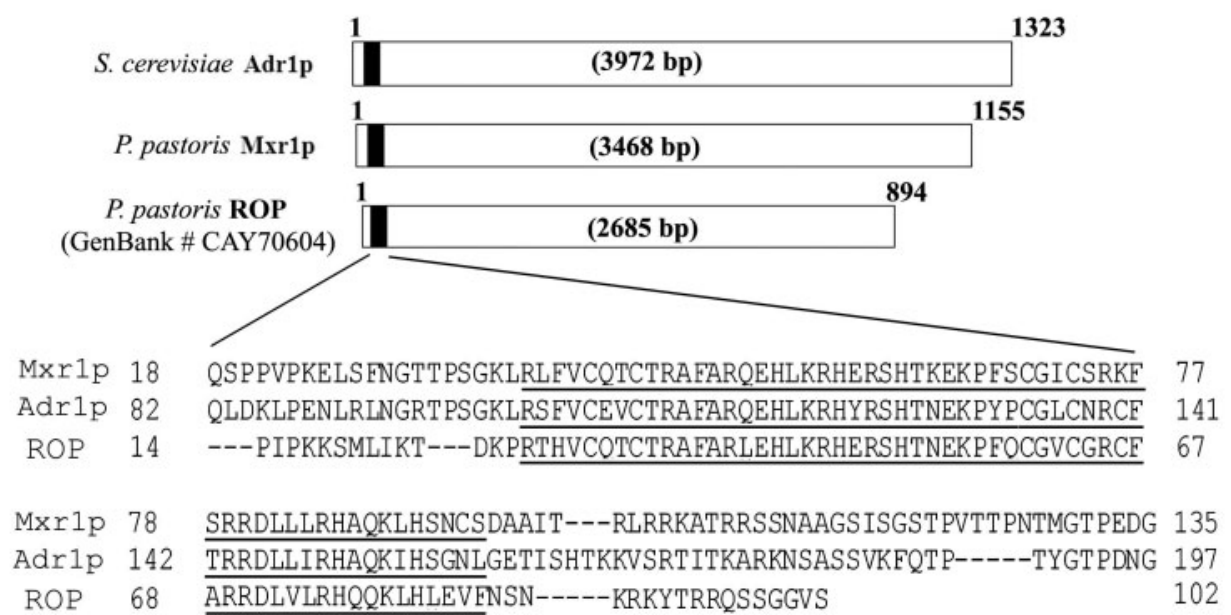

(b)

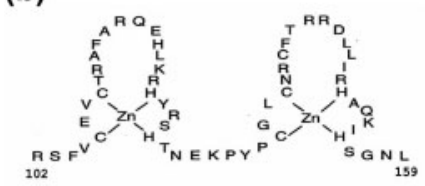

Adr1p

$\%$ identity (5'-CYCCNR-3')

(c)
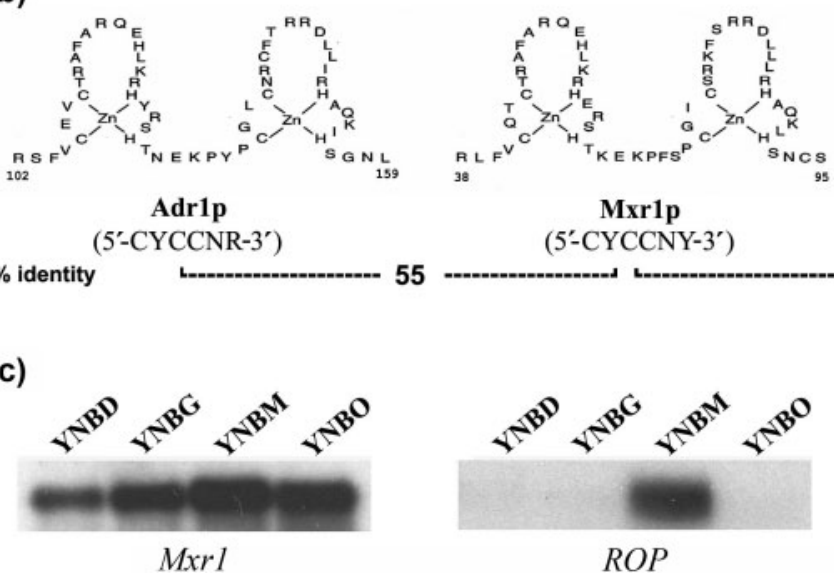

Mxr1p (5'-CYCCNY-3') 55
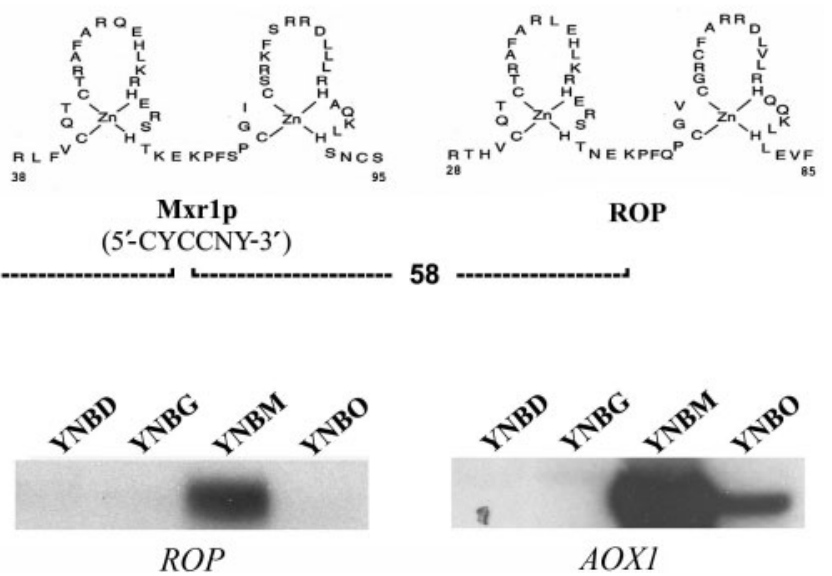

Fig. 1. Comparative analysis of zinc finger domains of ROP, Mxr1p and Adr1p, and analysis of ROP expression in $P$. pastoris. (a) Schematic representation of ORFs of Adr1p, Mxr1p and ROP and alignment of their amino acid sequences encompassing the N-terminal DNA-binding domain (black boxes). Numbers indicate amino acid residues. The length of each ORF is indicated in parentheses. Amino acids of the two zinc fingers are underlined. (b) Comparison of the putative $\mathrm{C}_{2} \mathrm{H}_{2}$ zinc finger domain of ROP with those of Adr1p and Mxr1p. The core nucleotide sequences essential for the binding of Adr1p and Mxr1p to DNA are shown in parentheses $(\mathrm{N}$, any nucleotide; $\mathrm{R}, \mathrm{C} / \mathrm{T} ; \mathrm{Y}, \mathrm{A} / \mathrm{G})$. The percentage amino acid sequence identity amongst the zinc fingers is indicated. (c) Northern blot analysis of Mxr1, ROP and AOXI.

2100 Bioanalyser (Agilent) as per the manufacturer's protocol. Total RNA purity was assessed by the NanoDrop ND-1000 UV-Vis Spectrophotometer (Nanodrop Technologies). Total RNA with $A_{260}: A_{280}>1.8$ and $A_{260}: A_{230} \geqslant 1.3$ was used for microarray experiments. RNA was considered to be good for use in microarray when the rRNA $28 \mathrm{~S}: 18 \mathrm{~S}$ ratios were $\geqslant 1.5$, with the rRNA contribution being $30 \%$ or more and the RNA integrity number (RIN) was $\geqslant 7.0$. RNA samples were labelled using the Agilent QuickAmp labelling kit ( $\mathrm{p} / \mathrm{n} 5190-0442)$. Five hundred nanograms of each of the control and test samples was incubated with reverse transcription mix at $40{ }^{\circ} \mathrm{C}$ and converted to double-stranded cDNA primed by oligo-dT with a T7 polymerase promoter. The cleaned-up double-stranded cDNAs were used as templates for complementary RNA (cRNA) generation. cRNA was generated by in vitro transcription and the dye Cy3 CTP (Agilent) was incorporated during this step. The cDNA synthesis and in vitro transcription steps were carried out at $40{ }^{\circ} \mathrm{C}$. Labelled cRNA was cleaned up and quality was assessed for yields and specific activity. The labelled cRNA samples were hybridized to the $P$. pastoris $8 \times 15 \mathrm{~K}$ microarray. Fragmentation of labelled cRNA and hybridization were done using the Gene Expression Hybridization kit from Agilent (Part Number 51885242). Hybridization was carried out in Agilent's Surehyb Chambers at $65{ }^{\circ} \mathrm{C}$ for $16 \mathrm{~h}$. The hybridized slides were washed using Agilent Gene Expression wash buffers (Part No: 5188-5327) and scanned using the Agilent Microarray Scanner G2505C at $5 \mu \mathrm{m}$ resolution. Data extraction from images was done using Feature Extraction software version 10.5.1.1 from Agilent. Feature-extracted data were analysed using GeneSpring GX Version 11 software from Agilent. Normalization of the data was done in GeneSpring GX using the percentile shift; percentile shift normalization is a global normalization, where the locations of all the spot intensities in an array are adjusted. This normalization takes each column in an experiment independently and computes the percentile of the expression values for this array, across all spots (where $n$ has a range from 0 to 100 and $n=75$ is the median). It subtracts this value from the expression value of each entity and normalizes these to specific samples. Genes whose 
expression was different by twofold and above were identified. Differentially regulated genes were clustered using hierarchical clustering to identify significant gene expression patterns.

\section{RESULTS}

\section{Analysis of $R O P$ expression and identification of ROP-regulated genes during methanol metabolism}

We recently demonstrated that Mxrlp, a master regulator of the Mut pathway in P. pastoris, regulates gene expression by binding to promoter sequences containing $5^{\prime}$-CYCCNY3'motif (Kranthi et al., 2009, 2010). The DNA-binding domain of Mxrlp shares $55 \%$ amino acid sequence identity with that of the $S$. cerevisiae zinc finger transcription factor Adrlp (Lin-Cereghino et al., 2006, Fig. 1), whose recognition sequence contains $5^{\prime}$-CYCCNR $-3^{\prime}$ rather than $5^{\prime}$ CYCCNY -3' motifs (Kranthi et al., 2009). A BLAST search of the $P$. pastoris genome sequence database with the amino acid sequence of Mxrlp led to the identification of a gene encoding a putative zinc finger protein (GenBank accession number CAY70604/XM_002492738.1). This protein, referred to as ROP (for repressor of PEPCK) in this study, was characterized in detail. The deduced amino acid sequence of ROP contains a putative $\mathrm{C}_{2} \mathrm{H}_{2}$ zinc finger domain, which shares $58 \%$ and $55 \%$ amino acid sequence identity with the zinc finger domains of Mxrlp and S. cerevisiae Adrlp respectively (Fig. 1a, b). While Mxr1 is constitutively expressed in $P$. pastoris cells cultured with different carbon sources (Lin-Cereghino et al., 2006, Fig. 1c), ROP expression was induced in $P$. pastoris cells cultured in a medium containing methanol (YNBM) but not glucose (YNBD), glycerol (YNBG) or oleic acid (YNBO) as the sole source of carbon (Fig. 1c). Methanol-inducible expression of $R O P$ as well as the significant levels of homology between the DNAbinding domains of ROP and Mxrlp led us to examine whether ROP has a role in the regulation of genes of the methanol utilization (Mut) pathway.

As a first step, we generated a $P$. pastoris strain $(\triangle R O P)$ in which the entire ORF of $R O P$ was replaced by a zeocin expression cassette (Fig. 2a). Deletion of the ROP coding region and loss of methanol-inducible expression of $R O P$ were confirmed by Southern and Northern analysis respectively (Fig. 2b, c). Northern analysis of Mxrlpregulated genes of the Mut pathway such as AOXI (alcohol oxidase I), FLDI (formaldehyde dehydrogenase I) and DHAS (dihydroxyacetone synthase) as well as peroxisomal genes such as PEX8 and PEX14 indicated that expression of these genes is not affected in $\triangle R O P$ (Fig. 2d). Furthermore, there was no significant difference in growth rates of WT (GS115) and $\triangle R O P$ strains cultured in YNBM medium (data not shown). To understand the physiological significance of methanol-inducible expression of ROP, transcripts which are expressed in WT and $\triangle R O P$ strains cultured in YNBM medium were hybridized to a $P$. pastoris microarray designed based on the $P$. pastoris genome (a)

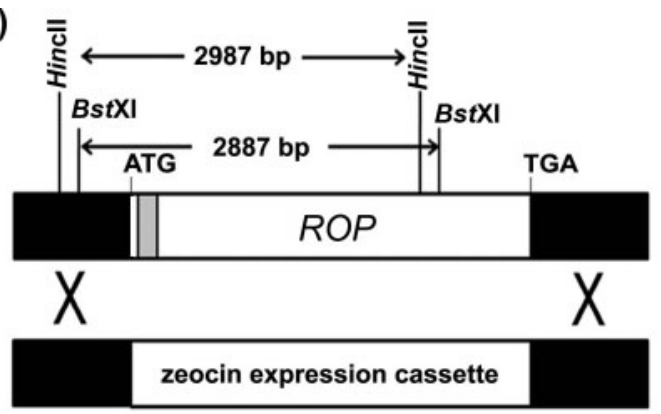

(b)

(c)
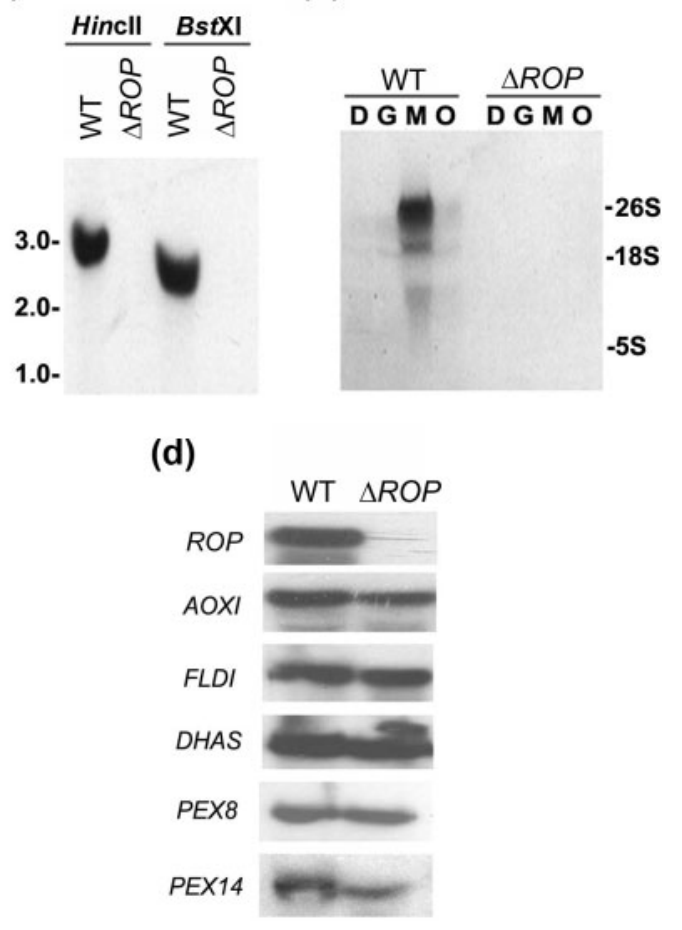

Fig. 2. Construction of $\triangle R O P$ and Northern analysis of genes involved in methanol metabolism. (a) Strategy for the generation of $\triangle R O P$. The coding region of $R O P$ was replaced by the zeocin expression cassette by homologous recombination (see Methods for details). Restriction enzymes used for confirmation of ROP deletion are indicated. The region corresponding to the DNAbinding domain is indicated by a grey box. (b) Southern analysis of Hincll- and BstXI-digested genomic DNA isolated from WT and $\triangle R O P$ strains. Fragments of $2987 \mathrm{bp}$ (Hincll) and $2887 \mathrm{bp}$ (BstXI) are generated in WT but not $\triangle R O P$, confirming deletion of the $R O P$ coding region. (c) Northern analysis of RNA isolated from WT and $\triangle R O P$ strains cultured in YNB medium containing glucose ('dextrose', D), glycerol (G), methanol (M) or oleic acid (O) as the sole source of carbon. The cDNA encoding the DNAbinding domain of ROP was radiolabelled and used to probe Southern and Northern blots. (d) Northern analysis of genes encoding enzymes of methanol metabolism and peroxins in WT and $\triangle R O P$ strains cultured in YNBM medium. 


\begin{tabular}{|c|c|c|}
\hline $6 \mathrm{~h}$ & & $24 \mathrm{~h}$ \\
\hline 5.05 & 1.Biotin synthase (CAY67102.1) * & 2.25 \\
\hline 3.77 & 2.Vitamin H Transporter (CAY 68553.1) * & 2.30 \\
\hline 3.74 & 3.Hypothetical protein (CAY69959.1) & 0.16 \\
\hline 2.60 & 4.Hypothetical protein (CAY67072.1) & -0.63 \\
\hline 1.80 & 5.Rho GTPase activating protein (CAY68534.1) & -0.34 \\
\hline 1.57 & 6.Hypothetical protein (CAY68618.1) & 1.41 \\
\hline 1.56 & 7.Hypothetical protein; Accession (CAY68609.1) & 0.33 \\
\hline 1.55 & 8.Catalytic subunit of mitochondrial inner membrane peptidase complex (CAY67311.1) * & 1.13 \\
\hline 1.42 & 9.Aspartic protease, attached to the plasma membrane via a GPI anchor (CAY70340.1) & -0.36 \\
\hline 1.40 & 10.SAM transporter of the mitochondrial inner membrane (CAY69962.1) * & 1.22 \\
\hline 1.39 & 11.Na+/Pi cotransporter, active in early growth phase (CAY68845.1) & -0.31 \\
\hline 1.38 & 12.Hypothetical protein (CAY71137.1) & -0.23 \\
\hline 1.38 & 13.Hypothetical protein (CAY67538.1) & -0.47 \\
\hline 0.94 & 14. Biotinapoprotein ligase (CAY70617.1) * & 1.34 \\
\hline 1.30 & 15.Putative protein of unknown function (CAY71758.1) & -0.11 \\
\hline 1.29 & 16. Hypothetical protein (CAY69933.1) & -1.64 \\
\hline 1.19 & 17.Delta (3,5)-Delta(2,4)-dienoyl-CoA isomerase, mitochondrial (CAY68552.1) * & 0.73 \\
\hline 1.12 & 18.Phytanoyl-CoA dioxygenase domain-containing protein (CAY68555.1) * & 1.39 \\
\hline 1.07 & 19.Protein of unknown function (CAY70008.1) & -0.38 \\
\hline 1.06 & 20. Hypothetical protein (CAY67877.1) & 0.18 \\
\hline 1.03 & 21.Fe(II)-dependent sulfonate/alpha-ketoglutarate dioxygenase & -0.74 \\
\hline 1.02 & 22. Hypothetical protein (CAY68619.1) & 0.65 \\
\hline 1.01 & 23. Hypothetical protein (CAY68223.1) & 1.11 \\
\hline-2.01 & 1.Hypothetical protein (CAY71232.1) & -1.15 \\
\hline-2.06 & 2.Hypothetical protein (CAY67579.1) & 0.49 \\
\hline-2.08 & 3.Hypothetical protein (CAY71232.1) & -1.14 \\
\hline-1.98 & 4.Hypothetical protein (CAY71232.1) & $-1,15$ \\
\hline-1.74 & 5.Hypothetical protein (CAY67499.1) & 0.19 \\
\hline-1.61 & 6. Hypothetical protein (CAY69960.1) & 0.40 \\
\hline-1.58 & 7.Hypothetical protein (CAY70746.1) & 0.30 \\
\hline-1.54 & 8.Hypothetical protein (CAY69943.1) & 0.06 \\
\hline-1.40 & 9.Hypothetical protein (CAY67309.1) & 0.18 \\
\hline-0.85 & 9.Hypothetical protein (CAY67309.1) & 0.73 \\
\hline-0.67 & 9.Hypothetical protein (CAY67309.1) & 0.69 \\
\hline-1.40 & 10. Hypothetical protein (CAY71604.1) & -0.26 \\
\hline-1.31 & 11.Hypothetical protein (CAY68739.1) & 0.25 \\
\hline-1.28 & 12. Transcriptional activator that enhances pseudohyphal growth (CAY71569.1) & 0.02 \\
\hline-1.26 & 13.Nitrilase (CAY68637.1) & -0.14 \\
\hline-1.05 & 14. Member of the $\mathrm{CCCH}$ zinc finger family (CAY71571.1) & 0.12 \\
\hline-1.22 & 15.Putative protein of unknown function (CAY71546.1) & 0.01 \\
\hline-1.20 & 16. Hypothetical protein (CAY71270.1) & -0.14 \\
\hline-1.18 & 17.Hypothetical protein (CAY70537.1) & -0.15 \\
\hline-1.16 & 18. Heme-activated, glucose-repressed Hap2p/3p/4p/5p CCAAT-binding complex (CAY71898.1) & -0.37 \\
\hline-1.24 & 19.Member of the CCCH zinc finger family (CAY71571.1) & 0.10 \\
\hline-1.10 & 20.Ferric reductase, reduces siderophore-bound iron uptake (CAY71469.1) & -0.22 \\
\hline-1.09 & 21.Subunit of an adoMet-dependent tRNA methyltransferase complex (CAY68219.1) & -0.75 \\
\hline-1.06 & 22.High-affinity cysteine-specific transporter with similarity to the Dal5p (CAY67069.1) & -0.12 \\
\hline-1.16 & 23.Member of the $\mathrm{CCCH}$ zinc finger family (CAY71571.1) & 0.21 \\
\hline-1.05 & 24.Ubiquitin-binding component of Rsp5p E3-ubiquitin ligase complex (CAY69915.1) & 0.94 \\
\hline-1.00 & 25.Low-affinity Fe(II) transporter of the plasma membrane (CAY71237.1) & -0.49 \\
\hline
\end{tabular}

Fig. 3. Heat map of genes upregulated or downregulated in $\triangle R O P$ cultured in YNBM medium for $6 \mathrm{~h}$ or $24 \mathrm{~h}$. Annotated genes which are upregulated at both $6 \mathrm{~h}$ and $12 \mathrm{~h}$ of growth are indicated by asterisks. sequence data available in GenBank (De Schutter et al., 2009). The results indicate that expression of several annotated as well as unannotated protein-coding genes is altered in $\triangle R O P$ grown in YNBM medium for $6 \mathrm{~h}$ or $24 \mathrm{~h}$ (Fig. 3). Expression of certain genes involved in biotin metabolism such as those encoding biotin synthase, vitamin $\mathrm{H}$ transporter and biotin-apoprotein ligase was increased in the $\triangle R O P$ strain cultured in YNBM medium for $6 \mathrm{~h}$ as well as $24 \mathrm{~h}$ (Fig. 3). Expression of genes of the Mut pathway was not affected in $\triangle R O P$, as observed by Northern analysis.

\section{ROP function in cells cultured under glucose- abundant, biotin-deficient conditions}

The activation of genes of biotin metabolism in the $\triangle R O P$ strain cultured in YNBM medium at both $6 \mathrm{~h}$ and $24 \mathrm{~h}$ led us to investigate whether ROP has a regulatory role in biotin metabolism. As a first step, we examined the growth of WT and $\triangle R O P$ strains in biotin-sufficient $\left(\mathrm{Bio}^{+}\right)$or biotin-deficient $\left(\mathrm{Bio}^{-}\right)$medium containing glucose and ammonium as the sole source of carbon and nitrogen respectively. While the WT strain could grow only in $\mathrm{Bio}^{+}$ 
medium, $\triangle R O P$ was able to grow in both $\mathrm{Bio}^{+}$and $\mathrm{Bio}^{-}$ media (Fig. 4a). Interestingly, ROP expression was induced in WT cells grown in Bio medium (Fig. $4 \mathrm{~b}$ ). The biotinindependent growth of $\triangle R O P$ is not due to ROP-mediated derepression of the de novo biotin biosynthethic pathway since genes encoding biotin synthase and biotin-apoprotein ligase were derepressed in the WT as well as the $\triangle R O P$ strain cultured in $\mathrm{Bio}^{-}$medium (Fig. 4c).

\section{Catabolite repression of PEPCK in $P$. pastoris cultured under biotin- and PC-deficient, glucose-abundant conditions}

Anaplerotic synthesis of OAA from phosphoenolpyruvate (PEP) is primarily catalysed by the biotin-dependent enzyme PC (Fig. 5a). It is known that aspartate can restore the growth of PC mutants in glucose-ammonium medium due to its conversion to OAA by transamination with

(a)

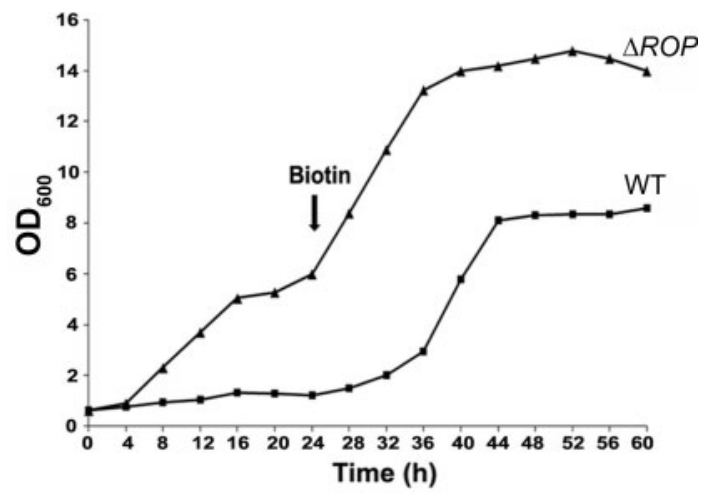

(b)

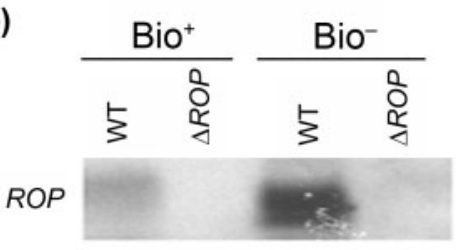

(c)

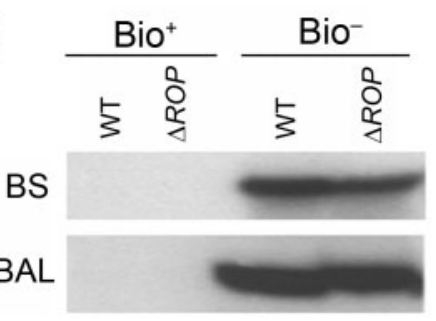

Fig. 4. Effect of biotin on $R O P$ expression and growth of $P$. pastoris. (a) Growth of WT and $\triangle R O P$ strains in $\mathrm{Bio}^{-}$medium. Biotin was added after $24 \mathrm{~h}$ of growth as indicated by the arrow. (b) Northern analysis of ROP in WT and $\triangle R O P$ cultured in $\mathrm{Bio}^{+}$ and $\mathrm{Bio}^{-}$medium. (c) Northern analysis of the genes encoding biotin synthase (BS) and biotin-apoprotein ligase (BAL) in WT and $\triangle R O P$ strains cultured in $\mathrm{Bio}^{+}$and $\mathrm{Bio}^{-}$medium. $\alpha$-ketoglutarate (Zelle et al., 2010; Blázquez et al., 1995). When aspartate was added to $\mathrm{Bio}^{-}$medium, growth of the WT strain was restored, indicating that the growth defect is primarily due to the deficiency of PC-mediated OAA synthesis (Fig. 5b). Furthermore, addition of phenylacetic acid (an inhibitor of PC) to $\mathrm{Bio}^{+}$cultures completely inhibited the growth of WT, but not $\triangle R O P$ (Fig. 5c). These results indicate that a PC-independent pathway of OAA synthesis (pyruvate carboxylase bypass) is responsible for the growth of $\triangle R O P$ in $\mathrm{Bio}^{-}$medium. Since enzymes of the

(a)

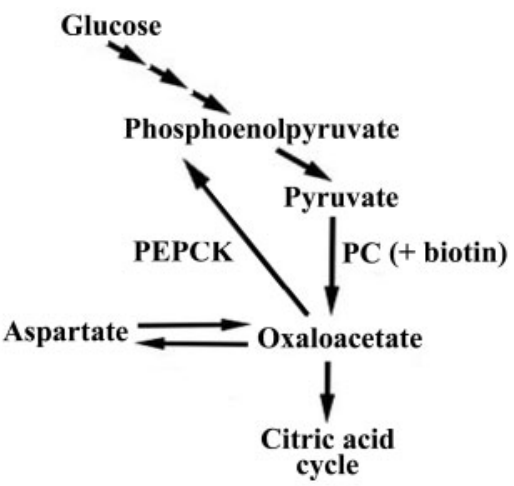

(b)

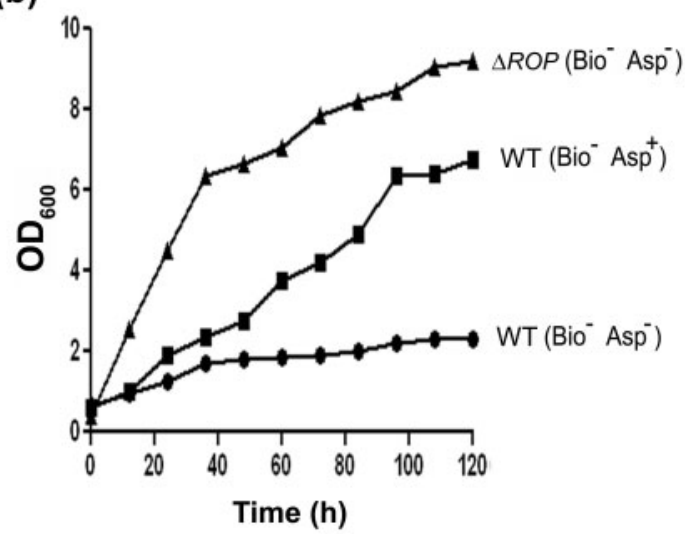

(c)

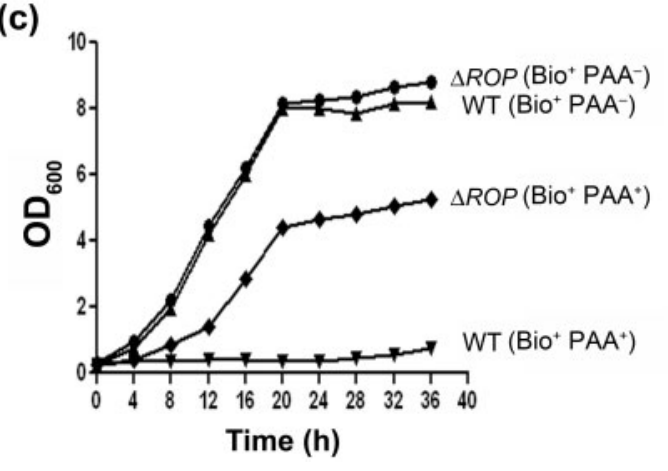

Fig. 5. Effect of aspartate and phenylacetic acid on the growth of $P$. pastoris. (a) Schematic representation of biosynthesis of OAA and PEP. (b) Effect of aspartate on the growth of WT and $\triangle R O P$ strains in $\mathrm{Bio}^{-}$medium. (c) Effect of phenylacetic acid (PAA) $(2.5 \mathrm{mM})$ on the growth of WT and $\triangle R O P$ in $\mathrm{Bio}^{+}$medium. 
glyoxylate cycle such as isocitrate lyase (ICL) and malate synthase (MS) as well as PEPCK are known to substitute for PC and contribute to OAA synthesis under specific growth conditions (Zelle et al., 2010; Blázquez et al., 1995). We examined their expression in WT and $\triangle R O P$ strains. Northern analysis indicated that biotin deficiency abolishes catabolite repression of ICL and MS in the WT as well as in $\triangle R O P$ (Fig. 6a). However, catabolite repression of PEPCK in Bio ${ }^{-}$cultures was abolished only in $\triangle R O P$ but not in the WT strain (Fig. 6a). Furthermore, PEPCK was derepressed by phenylacetic acid in $\mathrm{Bio}^{+}$cultures of $\triangle R O P$ but not the WT (Fig. 6b). Thus, inactivation of PC either by biotin deficiency or by PAA results in the derepression of PEPCK in $\triangle R O P$ but not the WT under glucose-abundant conditions. When methanol is the sole carbon source, derepression of PEPCK is observed in both the WT and $\triangle R O P$ (Fig. 6c). Thus, ROP is involved in the catabolite repression of $P E P C K$ specifically in glucose-abundant, biotin- and PC-deficient cultures of P. pastoris.

The DNA-binding domain of ROP shows significant homology to those of Adrlp and Mxr1 (Fig. 1). We reported earlier that Adrlp and Mxrlp bind to promoter sequences containing $5^{\prime}$-CYCCNY-3' and $5^{\prime}$-CYCCNR- $3^{\prime}$ respectively (Kranthi et al., 2009, 2010). In view of the high

(a)
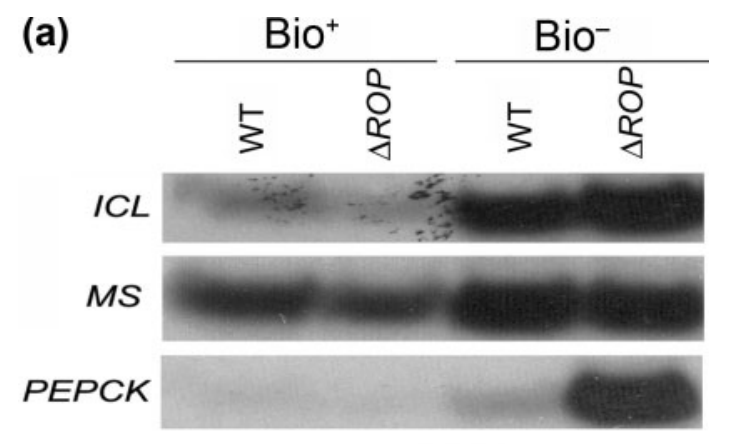

(b)

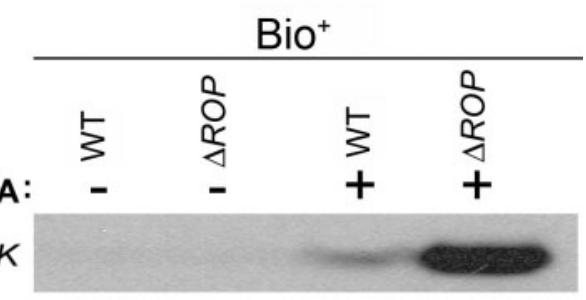

(c)

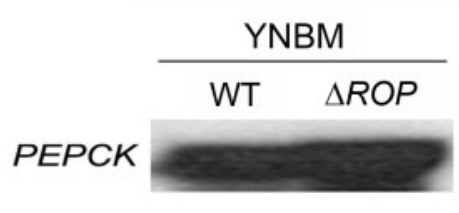

Fig. 6. Northern analysis of the genes encoding isocitrate lyase $(I C L)$, malate synthase $(M S)$ and PEPCK (PEPCK). (a) Expression of these genes in WT and $\triangle R O P$ strains cultured in $\mathrm{Bio}^{+}$and $\mathrm{Bio}^{-}$ medium. (b) Effect of PAA on PEPCK expression in WT and $\triangle R O P$ cultured in $\mathrm{Bio}^{+}$medium. (c) $P E P C K$ expression in WT and $\triangle R O P$ strains cultured in YNBM. degree of homology among the DNA-binding domains of ROP, Mxrlp and Adrlp (Fig. 1), it is likely that the DNAbinding specificity of ROP is similar to that of Adrlp or Mxrlp. To examine whether $5^{\prime}$-CYCCNY-3'/5'-CYCCNR$3^{\prime}$ is present in the PEPCK promoter, we carried out an in silico analysis and identified potential ROP-binding motifs in the PEPCK promoter (see Supplementary Fig. S1, available with the online version of this paper). Interestingly, ROP is also involved in the regulation of a number of genes other than PEPCK, as is evident from the microarray expression profiling of RNA isolated from $\mathrm{Bio}^{-}$cultures of $P$. pastoris (Supplementary Fig. S2). The results from the microarray were further validated by Northern analysis of selected genes (Supplementary Fig. S3). More studies are needed to understand the physiological significance of upregulation of these genes.

\section{DISCUSSION}

In this study, we have characterized a zinc finger protein named ROP whose expression is induced by methanol and by biotin and pyruvate carboxylase (PC) deficiency. Methanol-inducible expression of ROP results in the activation and repression of a number of genes including those involved in biotin metabolism. However, genes of the Mut pathway as well as peroxisomal genes which are regulated by Mxrlp are not the targets of ROP. The activation of genes of biotin metabolism such as biotin synthase and vitamin $\mathrm{H}$ transporter during methanol metabolism in the $\triangle R O P$ strain led us to investigate the role of ROP under biotin-deficient conditions. We demonstrate that ROP represses biotin-independent growth of $P$. pastoris. In glucose-abundant cultures, biotin deficiency results in the activation of $R O P$ and repression of PEPCK. Comparison of expression profiles of WT and $\triangle R O P$ strains indicates that ROP regulates the expression of several genes under biotin-deficient conditions. Since biotin deficiency inactivates PC, a key enzyme involved in the anaplerotic synthesis of OAA, we focused our attention on enzymes that are known to substitute for PC under certain growth conditions. We demonstrate that ROP has a key role in the derepression of PEPCK but not ICL and MS in biotin- and PC-deficient, glucose-abundant cultures of $P$. pastoris. The normal function of PEPCK is to catalyse the conversion of OAA to PEP. However, under certain conditions, PEPCK can catalyse the reverse reaction leading to the synthesis of OAA from PEP (Zelle et al., 2010; Blázquez et al., 1995). There is an excellent correlation between derepression of $P E P C K$ and biotin- and PC-independent growth of $\triangle R O P$. It is therefore tempting to speculate that PEPCK-catalysed OAA synthesis may have a key role in the biotin- and PCindependent growth of $\triangle R O P$. It is pertinent to note that OAA can also be synthesized in a biotin- and PCindependent manner via the glyoxylate cycle through ICL and MS. However, derepression of ICL and MS does not restore growth of the WT strain in $\mathrm{Bio}^{-}$medium. Thus, if OAA is synthesized via the glyoxylate cycle in $\mathrm{Bio}^{-}$cultures 
of $P$. pastoris, synthesis of PEP from OAA by PEPCK may be the growth-limiting reaction. Thus, either the gluconeogenic activity or the anaplerotic function of PEPCK may be responsible for the biotin- and PC-independent growth of $\triangle R O P$. More studies are needed to distinguish between these possibilities. ROP appears to be the first example of a yeast transcription factor that is specifically involved in catabolite repression of PEPCK under biotin- and PC-deficient conditions.

The phenotype of $\triangle R O P$ described in this study is similar to that of BPC mutants of S. cerevisiae (Blázquez et al., 1995). Both can grow in glucose-ammonium medium, despite PC deficiency. However, ICL is involved in overcoming PC deficiency in $S$. cerevisiae BPC mutants whereas PEPCK is involved in overcoming PC deficiency in $\triangle R O P$. While the transcription factors involved in the BPC phenotype are not known, we have demonstrated that ROP is responsible for the biotin- and PC-independent growth phenotype of $\triangle R O P$. Spontaneous mutants of PC-deficient P. pastoris strains, which can grow in glucose-ammonium medium, have been reported (Menéndez et al., 1998) and it will be interesting to examine $R O P$ expression in these mutants.

Although the physiological significance of $R O P$ expression during methanol metabolism is not clear at present, we wish to discuss an unusual function of PC during methanol metabolism which may be responsible for the activation of $R O P$ in methanol-grown cultures of $P$. pastoris. $P C$ is required for the assembly and peroxisomal import of alcohol oxidase, the first enzyme involved in methanol metabolism in methylotrophic yeasts such as $P$. pastoris and Hanseneula polymorpha (Ozimek et al., 2003, 2007). PC mediates flavin adenine dinucleotide (FAD) binding to AOX in the cytosol and this reaction is essential for its subsequent octamerization and import into peroxisomes. The transcarboxylation domain of PC, which participates in the transfer of the carboxyl group of carboxylated biotin to pyruvate, is involved in AOX assembly (Ozimek et al., 2003, 2007). Since AOX is the most abundant protein present in methanol-grown $P$. pastoris cells, it is possible that PC is primarily utilized for the assembly of AOX during methanol metabolism rather than for the synthesis of OAA. Thus, a decrease in the anaplerotic activity of PC, as observed in biotin- and $\mathrm{PC}$-deficient $\left(\mathrm{Bio}^{-}\right.$; $\mathrm{PAA}^{+}$, $\mathrm{Bio}^{+}$) cultures may occur during methanol metabolism as well. Thus, deficiency in the catalytic activity of PC may serve as a signal for the activation of ROP in $\mathrm{Bio}^{-}$as well as YNBM cultures. It should however be noted that ROP does not regulate PEPCK expression during methanol metabolism and its exact function during methanol metabolism is not clear at present. A detailed analysis of the function of ROP-regulated genes during methanol metabolism will be reported separately.

In summary, ROP is a novel methanol- and biotinstarvation-inducible zinc finger protein that represses the growth of $P$. pastoris under biotin- and PC-deficient, glucose-abundant conditions. ROP is specifically involved in catabolite repression of PEPCK and to our knowledge is the first yeast transcription factor that is involved in the catabolite repression of PEPCK under biotin- and PCdeficient conditions.

\section{ACKNOWLEDGEMENTS}

We thank G. Padmanaban for critical reading of the manuscript and suggestions. This work was funded by the Department of Biotechnology, New Delhi, India.

\section{REFERENCES}

Blázquez, M. A., Gamo, F. J. \& Gancedo, C. (1995). A mutation affecting carbon catabolite repression suppresses growth defects in pyruvate carboxylase mutants from Saccharomyces cerevisiae. FEBS Lett 377, 197-200.

De Schutter, K., Lin, Y. C., Tiels, P., Van Hecke, A., Glinka, S., WeberLehmann, J., Rouzé, P., Van de Peer, Y. \& Callewaert, N. (2009). Genome sequence of the recombinant protein production host Pichia pastoris. Nat Biotechnol 27, 561-566.

Gasser, B., Dragosits, M. \& Mattanovich, D. (2010). Engineering of biotin-prototrophy in Pichia pastoris for robust production processes. Metab Eng 12, 573-580.

Kim, H. S., Hoja, U., Stolz, J., Sauer, G. \& Schweizer, E. (2004). Identification of the tRNA-binding protein Arclp as a novel target of in vivo biotinylation in Saccharomyces cerevisiae. J Biol Chem 279, 42445-42452.

Kranthi, B. V., Kumar, R., Kumar, N. V., Rao, D. N. \& Rangarajan, P. N. (2009). Identification of key DNA elements involved in promoter recognition by Mxrlp, a master regulator of methanol utilization pathway in Pichia pastoris. Biochim Biophys Acta 1789, 460-468.

Kranthi, B. V., Vinod Kumar, H. R. \& Rangarajan, P. N. (2010). Identification of Mxrlp-binding sites in the promoters of genes encoding dihydroxyacetone synthase and peroxin 8 of the methylotrophic yeast Pichia pastoris. Yeast 27, 705-711.

Lin-Cereghino, G. P., Godfrey, L., de la Cruz, B. J., Johnson, S., Khuongsathiene, S., Tolstorukov, I., Yan, M., Lin-Cereghino, J., Veenhuis, M. \& other authors (2006). Mxrlp, a key regulator of the methanol utilization pathway and peroxisomal genes in Pichia pastoris. Mol Cell Biol 26, 883-897.

Maniatis, T., Fritsch, E. F. \& Sambrook, J. (1982). Molecular Cloning: a Laboratory Manual. Cold Spring Harbor, NY: Cold Spring Harbor Laboratory.

Menéndez, J., Delgado, J. \& Gancedo, C. (1998). Isolation of the Pichia pastoris PYC1 gene encoding pyruvate carboxylase and identification of a suppressor of the pyc phenotype. Yeast 14, 647654.

Ozimek, P., van Dijk, R., Latchev, K., Gancedo, C., Wang, D. Y., van der Klei, I. J. \& Veenhuis, M. (2003). Pyruvate carboxylase is an essential protein in the assembly of yeast peroxisomal oligomeric alcohol oxidase. Mol Biol Cell 14, 786-797.

Ozimek, P. Z., Klompmaker, S. H., Visser, N., Veenhuis, M. \& van der Klei, I. J. (2007). The transcarboxylase domain of pyruvate carboxylase is essential for assembly of the peroxisomal flavoenzyme alcohol oxidase. FEM Yeast Res 7, 1082-1092.

Phalip, V., Kuhn, I., Lemoine, Y. \& Jeltsch, J. M. (1999). Characterization of the biotin biosynthesis pathway in Saccharomyces 
cerevisiae and evidence for a cluster containing BIO5, a novel gene involved in vitamer uptake. Gene 232, 43-51.

Pirner, H. M. \& Stolz, J. (2006). Biotin sensing in Saccharomyces cerevisiae is mediated by a conserved DNA element and requires the activity of biotin-protein ligase. J Biol Chem 281, 12381-12389.

Schmitt, M. E., Brown, T. A. \& Trumpower, B. L. (1990). A rapid and simple method for preparation of RNA from Saccharomyces cerevisiae. Nucleic Acids Res 18, 3091-3092.
Wu, H., Ito, K. \& Shimoi, H. (2005). Identification and characterization of a novel biotin biosynthesis gene in Saccharomyces cerevisiae. Appl Environ Microbiol 71, 6845-6855.

Zelle, R. M., Trueheart, J., Harrison, J. C., Pronk, J. T. \& van Maris, A. J. (2010). Phosphoenolpyruvate carboxykinase as the sole anaplerotic enzyme in Saccharomyces cerevisiae. Appl Environ Microbiol 76, 53835389.

Edited by: R. P. Oliver 